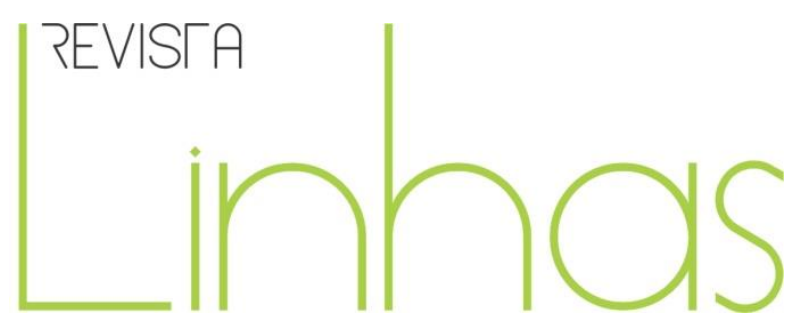

\title{
Per una storia dell'Università nelle raccolte museali: il Museo per la Storia dell'Università di Pavia e il Museo Europeo degli Studenti di Bologna
}

\begin{abstract}
Riassunti
Il presente contributo intende focalizzare la propria attenzione su una specifica tipologia di istituzione Universitaria, e cioè i musei per la storia dell'Università. In Italia, nonostante la tradizione ultrasecolare dell'Università, ne esistono solo due, il Museo per la Storia dell'Università di Pavia e il Museo Europeo degli Studenti di Bologna. Finora poco studiati, sono senza dubbio un esperimento riuscito di musealizzazione del patrimonio librario, fotografico e iconografico che documenta, celebra e valorizza la storia dell'Università.
\end{abstract}

Parole chiave: Storia dell'Università. Storia dell'Educazione. Storiografia. Storia degli studenti. Italia.

\author{
Luigiaurelio Pomante \\ Università degli Studi di Macerata \\ - Italia \\ luigiaurelio.pomante@unimc.it
}

\footnotetext{
Para citar este artigo:

POMANTE, Luigiaurelio. Per una storia dell'Università nelle raccolte museali: il Museo per la Storia dell'Università di Pavia e il Museo Europeo degli Studenti di Bologna. Revista Linhas. Florianópolis, v. 20, n. 44, p. 96-110, set./dez. 2019.
} 


\section{For a history of the Para uma história da University in museum universidade nas coleções de collections: the Museum for the History of the University of Pavia and the European Museum of Students in Bologna museus: o Museu para a história da Universidade de Pavia e o Museu europeu dos estudantes de Bolonha}

\begin{abstract}
The present article intends to analyze a specific type of university institution, i.e. museums for the history of the University. In Italy, despite the centuries-old university tradition, there are only two, the Museum for the History of the University of Pavia and the European Museum of Students (MEUS) in Bologna. So far little studied, they are undoubtedly a successful experiment of musealisation of the archival-library, photographic and iconographic heritage that documents, celebrate and valorize the history of the University.
\end{abstract}

Keywords: History of university. History of education. Historiography. History of students. Italy.

\section{Resumo}

A presente contribuição pretende centrar a sua atenção em um tipo específico de instituição universitária: os museus para a história da universidade. Na Itália, apesar da tradição ultrassecular da universidade, existem apenas dois: o Museu para a história da Universidade de Pavia e o Museu europeu dos estudantes de Bolonha. Pouco estudados até agora, não há dúvida de que sejam uma experiência bemsucedida de musealização do patrimônio livresco, fotográfico e iconográfico que documenta, celebra e valoriza a história da Universidade.

Palavras-chave: História da universidade. História da educação. Historiografia. História dos estudantes. Itália. 


\section{Introduzione}

Il dibattito storiografico sulle università e sull'istruzione superiore dell'ultimo decennio del Ventesimo secolo ha conosciuto in Italia una nuova e feconda stagione, grazie al profondo rinnovamento della storiografia di settore (MORETTI, 1996; POMANTE, 2017). Attraverso la costituzione di centri di ricerca locali altamente specializzati (BRIZZI, 1996) e soprattutto grazie alla nascita nel 1996 del Centro interuniversitario per la storia delle università italiane (CISUI), che per la prima volta ha raccolto studiosi di atenei diversi e di eterogenea provenienza disciplinare ma ugualmente interessati all'analisi delle fonti e all'approfondimento della storia delle università e dei sistemi d'istruzione superiore in Italia ed in Europa, è stato possibile registrare un notevole impulso nelle indagini e nel rinnovamento degli studi su questo versante. È pur vero, tuttavia, che la considerevole ed estremamente apprezzabile mole di studi prodotta, ha riservato una scarsa attenzione per una specifica tipologia di istituzione universitaria, ossia quella dei musei per la storia dell'Università, senza dubbio invece meritevoli di accurati e puntuali approfondimenti al pari dei musei della scuola e dell'educazione (MEDA, 2010; BRUNELLI, 2013; ASCENZIPATRIZI 2014; ANDREASSI-BARAUSSE-D’ALESSIO, 2016). Nello specifico appare opportuno soffermarsi in particolare sul Museo per la Storia dell'Università di Pavia e sul Museo Europeo degli Studenti (MEUS) di Bologna, strutture museali che, seppur molto distanti tra loro dal punto di vista tematico e sorte in momenti storici assai diversi, rappresentano attualmente gli unici due casi italiani di musei interamente dedicati alla storia universitaria. In queste due sedi, infatti, contrariamente a quanto non ancora accaduto in altri pur antichi e prestigiosi atenei italiani di lunga tradizione (si pensi, solo per citare alcuni casi, all'Università di Padova, di Roma o di Catania dove sono pur presenti importanti musei universitari ma non di storia dell'Università), è stato portato a termine un concreto ed esplicito progetto di musealizzazione del patrimonio archivistico-librario, fotografico, iconografico, ma anche e soprattutto oggettuale, che documenti e valorizzi la storia dell’Università. 


\section{Il Museo per la Storia dell’Università di Pavia}

Il Museo per la Storia dell'Università di Pavia (GALLIGARO, 1991; FREGONESE, 2001), ubicato all'interno del palazzo centrale universitario, affacciato sul cortile dei caduti, l'antico cortile medico dell'Università, affonda le radici della sua fondazione nel 1932, anno in cui la città pavese fu sede del IV Congresso della Società Italiana di Anatomia, organizzato in concomitanza con il centenario della morte di Antonio Scarpa, celebre anatomista che contribuì in maniera determinante alla grande stagione culturale di cui l'Università di Pavia fu protagonista tra la fine del Settecento e l'inizio dell'Ottocento (GUDERZO, 2003). Proprio in quell'occasione, il presidente del congresso, il prof. Antonio Pensa, docente di Anatomia umana normale, volle celebrare la memoria di Scarpa con una mostra, allestita presso Palazzo Botta, dei suoi cimeli originali (autografi, libri, strumenti chirurgici, preparati anatomici), scelti tra quelli presenti nelle varie collezioni universitarie. Al termine del congresso e della relativa mostra, fu l'allora rettore dell'Ateneo lombardo, il prof. Ottorino Rossi, a comprendere meglio di altri come fosse quanto mai opportuno, in piena sintonia con gli ideali fascisti del tempo (LA ROVERE, 2003; POMANTE, 2018), tentare il recupero della gloriosa tradizione universitaria italiana, favorendo la conoscenza e la valorizzazione dei tanti secoli di storia della sede pavese per mezzo di una struttura permanente che permettesse di conservare il patrimonio storicoculturale dell'Università e ne agevolasse la sua divulgazione presso un pubblico più ampio possibile. Di qui la scelta di istituire il Museo per la storia dell'Università di Pavia in alcune sale dell'antico Museo anatomico, ancora munite delle preziose scaffalature a muro fatte realizzare dallo Scarpa in perfetto stile veneziano. L'allestimento fu affidato allo stesso Pensa mentre per l'incarico di direttore della nascente struttura fu scelto il prof. Guido Sala, docente di Neurologia e appassionato bibliografo.

I pezzi della mostra costituirono dunque il nucleo iniziale intorno a cui il nuovo Museo si sviluppò. Sempre nello stesso anno affluirono alla nascente realtà museale anche numerosi oggetti storici che erano stati restituiti all'Università di Pavia dopo l'Esposizione di Storia della Scienza tenutasi a Firenze tra il maggio e l'ottobre del 1929, tra i quali diversi strumenti del Gabinetto di Fisica di Alessandro Volta (FREGONESE, 2001, p. 221). Ad arricchire ulteriormente la struttura provvidero sia Pensa che Sala i quali 
riuscirono a recuperare altro materiale medico proveniente da varie collezioni dell'Ateneo nonché dall'ospedale cittadino S. Matteo. Nel Museo confluirono così altri cimeli di Scarpa, preparati e carte del chirurgo e anatomista Giacomo Rezia e dall'anatomista Bartolomeo Panizza, oltre alla preziosa e considerevole raccolta medico-chirurgica accumulata dal chirurgo Luigi Porta nel corso della sua lunghissima e fruttuosa attività professionale.

Il Museo fu inaugurato ufficialmente nel 1936 e sin da subito fu ampliato grazie ad oggetti provenienti da svariati istituti universitari, da altri musei preesistenti o da donazioni private, conservando comunque la sua originaria vocazione prevalente per la Medicina e per la Fisica. La direzione della struttura museale fu affidata nel 1938 al prof. Pensa che mantenne il suo incarico fino alla morte (1970).

Durante la seconda guerra mondiale il Museo rimase chiuso ma già nell'immediato secondo dopoguerra, grazie al contributo appassionato e competente dell'allora rettore Plinio Fraccaro, il Museo beneficiò di un sensibile miglioramento degli spazi espositivi e accrebbe ulteriormente le sue collezioni. Il primo vero ed importante ampliamento degli spazi in dotazione al Museo si ebbe nel 1956, con l'annessione di un'ampia sala destinata all'esposizione di preziosi documenti cartacei attinenti allo sviluppo storico dell'Ateneo nel corso dei secoli mentre, sempre nello stesso periodo, in un'altra stanza, accanto ai cimeli voltiani già posseduti, furono accolti alcuni apparecchi fisici realizzati da Giuseppe Belli, secondo successore sulla cattedra voltiana di Fisica sperimentale. Intorno alla metà degli anni Sessanta, tuttavia, l'accresciuta notorietà della struttura pavese sull'intero territorio nazionale determinò l'incalzante confluire di nuove collezioni e nuovi pezzi, spesso anche molto eterogenei, fenomeno che, in un breve lasso di tempo, rese gli spazi a disposizione del Museo assolutamente esigui ed insoddisfacenti. Così, quando nel 1970 ad Antonio Pensa subentrò alla guida del Museo il prof. Bruno Zanobio, docente presso l'Ateneo lombardo di Storia della medicina, la prima preoccupazione del nuovo direttore, che sarebbe rimasto in carica per quindici anni, fu innanzitutto quella di garantire una riorganizzazione logistica della struttura; nel 1983 furono altresì completati i lavori riorganizzativi degli spazi espositivi, con il restauro delle scaffalature settecentesche originali e la distribuzione delle raccolte nelle tre sale dedicate alla Medicina ed in quella 
dedicata alla Fisica, contemperando il rispetto dei criteri che avevano ispirato nel tempo direttori e "sostenitori" del Museo con le necessarie esigenze di rinnovamento scientifico-tecnologico.

Oggi il Museo per la Storia dell’Università, diretto dalla prof.ssa Lidia Falomo e sempre più nucleo centrale del Sistema Museale dell'Ateneo di Pavia, conserva preparati anatomici e naturalistici, cimeli medico-biologici, strumenti di Fisica e chirurgici, manoscritti e testi a stampa, nonché documenti relativi alla storia plurisecolare dell'Ateneo, costituendo - come ebbe giustamente modo di osservare Luigi Belloni «uno dei sacrari più suggestivi della cultura Europea» (BELLONI, 1975, p. 151). Alla seconda metà del secolo XVIII - quando, a seguito delle riforme volute dall'imperatrice d'Austria Maria Teresa, l'Università di Pavia divenne uno centri scientifici più all'avanguardia in Europa - risalgono i nuclei originari delle collezioni principali: la sezione di Fisica, raccoltasi intorno agli strumenti dell'antico laboratorio di Alessandro Volta, e la sezione di Medicina, erede delle collezioni anatomiche che Antonio Scarpa aveva organizzato proprio nei locali che attualmente ospitano il Museo.

All'ingresso della struttura i visitatori sono accolti da una galleria di docenti illustri riconducibili all'Università di Pavia e alla sua invidiabile tradizione scientifica e culturale, rappresentati per mezzo di statue, stampe, busti, fotografie d'epoca e medaglioni realizzati in occasione dell'apertura del Museo. I ritratti presentati, distribuiti in maniera omogenea su tutte le pareti, permettono di ripercorrere idealmente e quasi per intero la storia dell'Ateneo di Pavia coprendo un arco temporale che va dal XV secolo fino agli anni Trenta del secolo scorso.

Dai locali dell'ingresso si accede quindi alle tre sale della sezione di Medicina (Sala Scarpa, Sala Porta, Sala Golgi) e alle due della sezione di Fisica (Sala Volta e il Gabinetto di Fisica dell'Ottocento).

La Sala Scarpa è dedicata ad Antonio Scarpa, medico e anatomista, allievo di Giambattista Morgagni, chiamato a Pavia per ricoprire la cattedra di Anatomia nel 1783, nel pieno della stagione di riforme che stavano trasformando l'Università in uno dei centri più all'avanguardia in Europa. Al suo interno si conservano dunque strumenti e preparati 
che rimandano all'attività della scuola anatomica da lui diretta. Allo stesso Scarpa ma soprattutto ai suoi successori, si pensi in particolare a Bartolomeo Panizza, sono inoltre riconducibili pezzi selezionati dalle collezioni anatomiche e anatomo-patologiche del suo Museo e alcune scatole di strumenti chirurgici donati a Scarpa dallo stesso Imperatore Giuseppe II. Altri pezzi esposti nella sala provengono da collezioni coeve a quella di Scarpa, come il Museo di Storia Naturale di Lazzaro Spallanzani (1729-1799) e alludono al più generale contesto scientifico-culturale dell'epoca.

Ai progressi nel campo della Chirurgia e della Clinica attraverso i secoli XIX e XX, è invece dedicata la Sala Porta, intitolata appunto al già ricordato chirurgo ottocentesco Luigi Porta. Essa ospita preparati e reperti, protocolli di esperienze e cartelle cliniche compilate proprio da Porta e provenienti dal Museo che il chirurgo pavese aveva allestito nella clinica chirurgica dell'Ospedale San Matteo, poi, come visto, inglobato nel Museo per la Storia dell'Università. Nella sala è altresì conservato ed esposto anche il decreto di Napoleone del 23 giugno 1800 nel quale si fornivano istruzioni precise e puntuali per la riorganizzazione dell'Ateneo pavese nelle tre facoltà di Filosofia, Medicina e Giurisprudenza.

Nell'ultima sala della sezione di Medicina, infine, la Sala Golgi, ad essere protagonista è l'attività della scuola scientifica fondata da Camillo Golgi, docente a Pavia di Istologia e Patologia generale, ma soprattutto primo italiano a vincere nel 1906 il premio Nobel per la Medicina grazie alla messa a punto di un metodo istologico che pose le basi delle moderne neuroscienze. Nella sala sono conservati documenti autografi, preparati microscopici, disegni, strumenti nonché l'attestato del premio Nobel, vinto ex aequo con il suo antagonista, lo scienziato spagnolo Santiago Ramón y Cajal. Nella stessa sala si "custodisce" poi la testimonianza di altri scienziati che si formarono alla scuola pavese, quali ad esempio Paolo Mantegazza, Eusebio Oehl, Carlo Forlanini, Adelchi Negri.

A costituire la sezione di Fisica del Museo per la Storia dell'Università di Pavia sono invece il Gabinetto di Fisica di Alessandro Volta (Sala Volta), che insegnò Fisica sperimentale a Pavia a partire dal 1778, e il ricostruito Gabinetto di Fisica dell’Ottocento. Nella prima sala sono conservate le invenzioni di Volta e gli strumenti da lui utilizzati per ricerca e didattica mentre nella seconda sono esposti gli strumenti inventati o utilizzati dai 
successori alla cattedra di Fisica nel corso dell'800. La Sala Volta, inaugurata nel 1999, in occasione delle celebrazioni per il bicentenario dell'invenzione della pila, ospita una pregiata collezione di strumenti appartenenti all'originario gabinetto voltiano. Su un lato della sala, due tavoli da lavoro appartenenti a Volta ospitano alcuni esemplari dei numerosi strumenti (elettrofori, elettroscopi a pagliuzze ed elettroscopi condensatori, elettrometri, scaricatori, ecc.) che egli utilizzò per investigare le proprietà della carica elettrica e dei corpi elettrizzati, nonché alcune copie della pila di Volta. Al centro della sala, due vetrine contengono strumenti di meccanica e di pneumatica appartenenti al liceo Ugo Foscolo di Pavia: apparecchi per lo studio del moto su di un piano inclinato e per lo studio degli urti elastici, pompe, una fontana intermittente e un apparecchio per valutare la resistenza dell'aria. Si tratta di strumenti acquistati o fatti costruire da Volta e che furono trasferiti intorno alla metà del XIX secolo quando una riforma degli ordinamenti scolastici assegnò ai licei l’insegnamento della Meccanica.

Il Gabinetto di Fisica dell'Ottocento, restaurato e aperto al pubblico, ospita invece gli strumenti raccolti dai successori di Alessandro Volta nella cattedra di Fisica dell'Ateneo pavese. Si tratta di una collezione estremamente ricca (circa 600 strumenti, alcuni dei quali pezzi unici al mondo), a conferma di come le attività di ricerca e di didattica in Fisica sperimentale si confermarono particolarmente intense anche dopo la morte del fisico comasco. In particolare, Giuseppe Belli, che diresse il Gabinetto intorno alla metà del XIX secolo, arricchì notevolmente la collezione, anche con diversi apparecchi di sua invenzione. Tra questi è possibile oggi apprezzare il generatore elettrostatico ad induzione (che lui chiamò «ad attuazione»), un elettrometro di Bohnenberger da lui modificato, un motore magneto-elettrico e il suo famoso duplicatore. Da segnalare, infine, anche la presenza di un apparecchio per lo studio e la cura delle patologie polmonari di Carlo Forlanini.

Ad arricchire il posseduto del Museo per la Storia dell'Università di Pavia, una struttura museale a carattere storico-medico fatta di personaggi e discipline che hanno permesso alla sede pavese di divenire nei secoli un punto di riferimento imprescindibile nel panorama universitario italiano, concorre altresì la presenza di un annesso archivio del Museo che conserva documentazione storica a partire dal XV secolo e che costituisce una 
considerevole integrazione di quanto conservato all'Archivio di Stato di Pavia (antico archivio dell'Università) e all'Archivio storico dell'Università.

Appare opportuno rilevare in questa sede che le collezioni conservate nel Museo di Pavia nacquero soprattutto come supporto alla didattica e alla ricerca, attività che ancora oggi continuano a rivestire un ruolo di centrale importanza. Non a caso il Museo pavese, oltre a dedicare gran parte delle proprie energie al possibile incremento delle collezioni museali e a sviluppare interessanti e innovativi progetti culturali e multimediali, quali ad esempio il Pavia Project Physics, promosso dal prof. Fabio Bevilacqua e che ha dato vita alla creazione di un portale interamente dedicato alla valorizzazione e alla diffusione dell'imponente patrimonio storico-scientifico accumulatosi nell'Università di Pavia nel corso della sua gloriosa e plurisecolare storia (BEVILACQUA, 1998), è impegnato quotidianamente nella collaborazione con scuole di ogni ordine e grado per visite, attività e laboratori didattici. Con cadenza annuale, infine, il Museo partecipa alla realizzazione di mostre organizzate dal Sistema Museale di Ateneo per diffondere presso il grande pubblico la conoscenza del prezioso patrimonio dell’Università di Pavia: narrazioni espositive dedicate alle grandi scoperte realizzate nel corso dei secoli o a docenti illustri della sede pavese che hanno contribuito in maniera determinante a "fare" la storia della scienza.

\section{Il Museo Europeo degli Studenti (MEUS) di Bologna}

Dal carattere meno istituzionale, di più recente costituzione e tematicamente più «innovativo» rispetto a quello pavese, il Museo Europeo degli Studenti (MEUS) di Bologna rappresenta un vero e proprio unicum nel panorama museale europeo (BRIZZI, 2008; CAVALIERI, 2009; BRIZZI, 2010). Esso, infatti, intende favorire la conoscenza e lo studio del mondo studentesco ripercorrendo, attraverso oltre otto secoli, il ruolo che lo studente ha ricoperto all'interno dell'Università e della società. Proprio per riconoscere a questo particolare protagonista della vita accademica «quel rispetto che merita come elemento fondamentale del mondo universitario» (CAVALIERI, 2009), nel marzo del 2009 l'Alma Mater Studiorum di Bologna, ateneo tra i più antichi in Europa e sede per 
eccellenza delle prime associazioni studentesche, le universitates, ha inaugurato tale realtà museale con il preciso obiettivo di favorire la conoscenza dei mutamenti inerenti la figura dello studente nel corso dei secoli, attraverso l'illustrazione dei diversi aspetti che ne hanno connotato gli sviluppi nei vari Paesi europei: la mobilità studentesca, la vita materiale, l'associazionismo, l'impegno politico, le attività goliardiche e sportive, la tardiva ammissione delle donne nelle università, gli aspetti originali della cultura studentesca (giornali, musica, teatro, feste).

La nascita e lo sviluppo di tale importante realtà museale, dedicata alla storia di quegli studenti che, oltre otto secoli fa, proprio a Bologna si diedero comuni regole di convivenza e autogoverno, ideando rituali e cerimoniali divenuti elementi fondanti della loro identità, devono essere necessariamente ricollegati ad un preciso ed interessante progetto nato ormai oltre venti anni fa dall'incontro fra alcuni storici che studiavano la presenza studentesca nelle università, diretti dal prof. Gian Paolo Brizzi (allora docente di Storia moderna e deus ex machina del nascente e già ricordato CISUI) e alcuni esponenti di associazioni di ex studenti, sollecitati da Marco Bortolotti (a quel tempo direttore dell'Archivio Storico), spinti a donare i cimeli della loro esperienza da studenti. Il progetto, almeno nella sua fase iniziale, mirava ad una semplice esposizione di oggetti delle associazioni goliardiche ma, dopo una prima mostra (GAUDEAMUS IGITUR, 1995), come ha avuto modo di illustrare lo stesso Brizzi

maturò un intento più ambizioso, capace di rappresentare - in forma necessariamente sintetica - la storia degli studenti universitari europei dalle origini ad oggi. Promuovemmo pertanto seminari, convegni, incontri di studio - confluiti poi in volumi miscellanei, monografie, atti di convegno - per interrogarci su cosa rappresenti la storia degli studenti per lo storico delle mentalità collettive, per il politologo, per lo studioso di processi di formazione delle classi dirigenti, per lo storico del costume e della società. (Brizzi, 2005, p. 5-7)

Ovviamente tale iniziativa, pur stimolante, dovette i conti con il giudizio non sempre lusinghiero da parte del "conservatore" mondo accademico nei confronti della componente studentesca e delle sue specifiche peculiarità e soprattutto costrinse gli 
ideatori del progetto ad interrogarsi in via preliminare proprio sull'opportunità o meno di ricorrere alla formula museale quale contenitore ideale per raccogliere il patrimonio della vita studentesca.

\begin{abstract}
L'adozione del termine 'museo' - ha spiegato ancora Brizzi - richiama nel nostro comune sentire l'idea di qualcosa che ha assunto un assetto stabile, definitivo nelle nostre conoscenze e nella funzione che gli è assegnata nel campo del sapere. La condizione di studente è invece una fase di passaggio nella vita di un individuo, è un'età della vita che lascia alle spalle ben poche scorie utili ad un'esposizione museale; [...] Forse [proprio per questo] potevano essere più adatti i termini di laboratorio di ricerca, di centro di documentazione ma considerammo che il termine museo sarebbe servito a riconoscere alla figura dello studente quel rispetto che merita come componente centrale del mondo universitario. (Brizzi, 2005, p. 7-8)
\end{abstract}

Di qui, la scelta condivisa di individuare quale sede della nuova realtà museale proprio la città di Bologna, luogo dove per la prima volta nella storia gli studenti si diedero una struttura associativa indipendente, capace di autogovernarsi attraverso l'elezione periodica di rettori, anch'essi studenti, che rimasero per secoli l'autorità preminente dello Studium. Tale studente sarebbe appunto divenuto nel 2009 il protagonista indiscusso del Museo Europeo degli Studenti di Bologna, soddisfacente coronamento degli sforzi compiuti in tal senso da Brizzi e dai suoi colleghi.

Ospitato al secondo piano di Palazzo Poggi, nel cuore del complesso universitario bolognese di via Zamboni, il MEUS illustra dunque la storia della presenza e delle tradizioni studentesche nelle principali città universitarie europee dalle origini ad oggi, attraverso materiale proveniente da antichi fondi archivistici, donazioni o acquisizioni presso antiquari. Il suo patrimonio, costituito da testimonianze della vita studentesca che si dipanano lungo un arco cronologico che va dal XIII secolo fino al 1968 e oltre e che continua ad arricchirsi periodicamente grazie alle donazioni provenienti da ogni città europea da parte di enti pubblici e di soggetti privati, è formato da un corpus selezionato di "testimonianze studentesche" costituito da fotografie, sculture, dipinti, diplomi, pergamene, editti, medaglie, onorificenze, cimeli di vario genere, libretti universitari, programmi di feste goliardiche, cartoline, manifesti, oltre che naturalmente da libri, e da 
circa duecento periodici. Ad arricchire ulteriormente il posseduto contribuisce altresì un database contenente i dati di oltre 450.000 studenti che si sono laureati a Bologna tra il 1380 e i giorni nostri.

Appare impossibile in tale sede descrivere analiticamente l'ingente materiale raccolto in questi anni dai curatori della collezione museale bolognese ma è comunque opportuno sottolineare come ogni gruppo studentesco ed ogni generazione abbia mirato nelle diverse epoche a produrre propri simboli e propri segni distintivi, riproposti oggi all'interno del museo di Via Zamboni attraverso distinte sezioni che ripercorrono la vita dello studente, l'evoluzione del suo status, le sue mutevoli identità ma anche la storia delle associazioni studentesche e i loro non sempre costanti rapporti con l'istituzione universitaria. II MEUS è articolato dunque attraverso un percorso suddiviso in cinque specifiche sezioni, dedicate alla vita quotidiana e alla disciplina intellettuale e fisica, all'ingresso delle donne all'Università, alla partecipazione politica degli studenti, alla goliardia ed alle tradizioni folkloristiche studentesche.

La prima sezione (denominata I caratteri originari) intende ripercorrere alcuni degli aspetti salienti della costituzione di questa nuova figura dello studente a partire dalla presentazione del privilegio di Federico I del 1155, fondamento di ogni successiva disposizione a tutela dello studente del quale, si ripercorrono, con una serie di apposite vetrine a tema, le forme organizzative dell'autonomia corporativa, l'organizzazione dei gruppi (o nazioni) e delle università studentesche, i rituali di ammissione, le pratiche devozionali e, non da ultima, la peregrinatio academica. La seconda sezione (Disciplinare i comportamenti, gli intelletti, i corpi) descrive invece i mutamenti verificatisi a partire dalla fine del medioevo nel ruolo assunto dallo studente sia in rapporto all'istituzione che alle attese della società e del potere politico che intendevano esercitare il proprio controllo sulla formazione delle necessarie competenze alla futura classe dirigente. In età moderna, fra il XVI e il XIX secolo, il mondo studentesco, superato l'impianto corporativistico dei secoli passati, finisce per frantumarsi in una miriade di piccole associazioni, ciascuna gelosa custode della propria identità regionale. Si affermano così nuovi rituali che valorizzano il genere e il carattere elitario del mondo studentesco, inserendo anche lo sport come parte integrante della formazione mentre l'ideologia meritocratica riesce ad 
imporsi come un valore chiamato a modellare il comportamento dello studente. A caratterizzare la terza sezione (La donna all'Università) è il riuscito tentativo di ripercorrere la storia dell'ingresso della donna all'Università, in un processo lento, graduale, difficoltoso, ma che alla fine ha determinato il mutamento dell'identità del mondo studentesco e delle forme della sociabilità. La quarta sezione (L'impegno politico) illustra l'impegno politico del giovane nel corso degli ultimi due secoli, quando, con il tramonto della società di Antico Regime, si esaurisce definitivamente la condizione privilegiata dello studente. Egli diventa, come ogni altro coetaneo, un cittadino e in quanto tale è chiamato a partecipare attivamente alla vita della propria nazione e a servire in armi la propria patria. Di qui il coinvolgimento nell'azione politica anche nelle forme di un impegno militare diretto: dai battaglioni studenteschi attivi in età napoleonica o nelle battaglie risorgimentali in Italia o Germania come in Grecia, alla partecipazione politica a sostegno o in opposizione ai movimenti totalitari fino alle lotte studentesche degli anni della contestazione sessantottina. La quinta ed ultima sezione (Cultura e folklore studentesco), infine, si sofferma sulle attività, in prevalenza a carattere ludico, promosse dalle tradizionali organizzazioni studentesche europee: dai giornali degli studenti alle piéces teatrali, dai manifesti fino agli abbigliamenti e ai copricapo tradizionali, senza tralasciare addirittura alcuni filmati dei tradizionali carnevali studenteschi che completano nel migliore dei modi questo affascinante ed innovativo percorso museale allestito dal MEUS di Bologna. Nel concreto e non vano auspicio che presto anche altre sedi universitarie si impegnino a "riscoprire" la propria tradizione e la propria gloriosa storia, allestendo, magari grazie al determinante contributo di qualche "illuminato" rettore, nuovi musei per la storia dell'Università, al fine di recuperare e valorizzare quell'ingente patrimonio storico-artistico appartenente alla migliore tradizione universitaria italiana, ma oggi purtroppo mal conservato in uno dei tanti abbandonati e polverosi archivi della Penisola o, peggio ancora, dimenticato con colpevole negligenza in qualche dismesso e fatiscente magazzino universitario. 


\section{Riferimenti}

ANDREASSI, Rossella; BARAUSSE, Alberto; D’ALESSIO, Michela. Museo della scuola e dell'educazione popolare dell'Università degli Studi del Molise. Cabás. Polanco: Crieme, v. 16, p. 143-167, 2016.

ASCENZI, Anna; PATRIZI, Elisabetta. I Musei della scuola e dell'educazione e il patrimonio storico-educativo. Una discussione a partire dall'esperienza del Museo della scuola «Paolo e Ornella Ricca» dell'Università degli Studi di Macerata. History of Education \& Children's Literature. Macerata: Eum, v. 9, n. 2, p. 685-714, 2014.

BEVILACQUA, Fabio. Pavia ProjectPhysics, Annali di storia delle università italiane. Bologna: CISUI, v. 2, p. 306-307, 1998.

BRIZZI, Gian Paolo. La storia delle università in Italia: l'organizzazione della ricerca nel XX secolo. In: SITRAN REA, Luciana (a cura di). La storia delle università italiane. Archivi, fonti, indirizzi di ricerca. Atti del convegno. Padova, 27-29 ottobre 1994. Trieste: Edizioni Lint, 1996, p. 273-309.

BRIZZI, Gian Paolo. Nasce Meus. Un nuovo museo per l'Alma Mater: questa volta si parla di studenti. A.D. 1088. Rivista dell’Università di Bologna. Bologna: Bologna University Press, v. 1, p. 5-14, 2008.

BRIZZI, Gian Paolo. Un Museo dedicado a los estudiantes de las universidades europeas. In PESET REIG, Mariano (pr.). Facultates y grados. X Congreso Internacional de historia de las Universidades Hispánicas (Valencia, Noviembre 2007). Valencia: Universitat de València, 2010, v. I, p. 275-284.

BRUNELLI, Marta. La catalogazione dei «beni culturali» della scuola: questioni metodologiche e concettuali. In: CAVALLERA, Hervé Antonio (a cura di). La ricerca storico-educativa oggi. Un confronto da metodi, modelli e programmi di ricerca. LecceBrescia: Pensa MultiMedia, 2013, v. I, p. 193-218.

CAVALIERI, Raffaella. Tra storia delle università e viaggio d'istruzione: l'evoluzione degli atenei europei riprodotta a Bologna nel primo Museo Europeo degli Studenti. Astrolabe, Clermond: Centre de Recerche sur la Literature des Voyages, v. 5, n. 28, 2009, on line.

FREGONESE, Lucio. Il Museo per la storia dell'Università di Pavia: storia, patrimonio e nuovi allestimenti. Annali di storia delle università italiane. Bologna: CISUI, v. 5, p 221-226, 2001.

GALLIGARO, Alberto. II museo per la storia dell'Università di Pavia. Politecnico: rivista del Politecnico di Milano. Milano: University Press, v. 4, p. 6-11, 1991. 
Gaudeamus igitur: studenti e goliardia 1888-1923. Mostra tenuta a Bologna nel 1995. Bologna: Bologna University Press, 1995.

GUDERZO, Giulio (a cura di). Per una storia dell'Università di Pavia. Bologna, CLUEB, 2003.

LA ROVERE, Luca. Storia dei Guf: organizzazione, politica e miti della gioventù universitaria fascista, 1919-1943, Torino: Bollati Boringhieri, 2003.

MEDA, Juri. Musei della scuola e dell'educazione. Ipotesi progettuale per una sistematizzazione delle iniziative di raccolta, conservazione e valorizzazione dei beni culturali delle scuole. History of Education \& Children's Literature. Macerata: Eum, v. 5, n. 2, p. 489-501, 2010).

MORETTI, Mauro. La storia dell'Università italiana in età contemporanea. Ricerche e prospettive. In: SITRAN REA, Luciana (a cura di). La storia delle università italiane. Archivi, fonti, indirizzi di ricerca. Atti del convegno. Padova, 27-29 ottobre 1994. Trieste: Edizioni Lint, 1996, p. 335-381.

MUSEO PER LA STORIA DELL'UNIVERSITÀ DI PAVIA. Attualità in storia della medicina e storia della fisica. Omaggio a Bruno Zanobio. Pavia: Goliardica Pavese, 1996.

PENSA, Antonio. Visita al Museo della storia dell'Università di Pavia, Milano: Alfieri e Lacroix, 1961.

POMANTE, Luigiaurelio. Las investigaciones sobre la historia de las universidades en Italia. Un balance historiográfico del siglo pasado. CIAN-Revista de Historia de las Universidades. Madrid: Editorial Dykinson, v. 20, n. 1, p. 163-192, 2017.

POMANTE, Luigiaurelio. Giuseppe Bottai e il rinnovamento fascista dell'Università italiana (1936-1942), Milano: FrancoAngeli, 2018.

Recebido em: 21/03/2019 Aprovado em: 11/06/2019

Universidade do Estado de Santa Catarina - UDESC Programa de Pós-Graduação em Educação - PPGE Revista Linhas

Volume 20 - Número 44 - Ano 2019 revistalinhas@gmail.com 